\title{
Designing Technology for Older People - The Role of Technical Self-confidence in Usability of an Inclusive Heating Control
}

\author{
Nicola Combe ${ }^{*}$, David Harrison, and Hua Dong \\ School of Engineering and Design, Brunel University, Uxbridge, UB8 3PH \\ niccombelgmail.com
}

\begin{abstract}
The ageing population of the UK is providing a large market opportunity for inclusive products and services. Yet older people are often excluded from using new technology due to inadequate consideration of their needs during the design process. This study focused specifically on including older people (aged 50-80) in the testing of a novel heating control interface under development. Recent studies have used two scalar methods to assess self-confidence; building upon this a technical self-confidence questionnaire was developed and completed by participants prior to attempting a usability task using the prototype. This study found that high technical self-confidence was inversely correlated to successful task performance. The participants who rated themselves as most technically self-confident were not successful in completing the task. Whereas, participants that rated themselves less confident had greater success completing the task. In general older people reported high levels of technical self-confidence and they were found to be willing to engage with the technical prototype. This highlights the high expectations of the older users group to be able to effectively engage with new technological systems. Designers should aim to instil further confidence amongst older users and provide systems that both support and include older people.
\end{abstract}

Keywords: Inclusive design, usability testing, older people, technical selfconfidence, technology.

\section{Introduction}

Inclusive interaction design has become increasingly significant in recent years, specifically in relation to product and mobile interfaces. In 2010 Langdon and Thimbleby introduced a special issue of Interacting with Computers with a discussion of the main theme 'Inclusion and Interaction'. The need for such a special issue was highlighted by the fact that although designing products for customers the 'user' is often not defined specifically. This can lead to designers basing their work on their

\footnotetext{
* Corresponding author.
} 
own, somewhat limited, experiences (Langdon and Thimbleby, 2010). The main criticism of existing usability studies is the common focus on testing in laboratory settings using only student participants, often under 30 years old.

Designing technology for older people can present unique challenges due to declines in motor, cognitive and sensory function associated with the ageing process. This paper presents a usability study, which examined whether older people (aged 5080 ) were successful in interacting with a prototype heating control interface and whether their reported technical self-confidence influenced success.

As Coleman (2003) argues that an accessible product is not necessarily inclusive, similarly Abascal and Nicolle argue, "even if the services are accessible...it is also important that users can perform those tasks easily, effectively and efficiently" (pp. $486,2005)$ through the user interface. Langdon and Thimbleby (2010) concluded that there is a need for increased knowledge transfer from inclusive design to the humancomputer interaction (HCI) community to help design more inclusive interactions.

Several studies have recognised that older users may have difficulties interacting with heating controls and energy management interface (Combe et al., 2012, Meier et al., 2011, Caird and Roy, 2008, and Freundenthal and Mook 2003). The realisation that older users in particular may struggle to use product interfaces was also recognised by Zhang, Rau and Salvendy (2009) and Sauer, Wastell and Schmeink (2009). When using a smart home interface to control a range of energy consuming activities within the home, older users took longer to complete tasks and made more errors than younger users (Zhang, Rau and Salvendy 2009).

Recent studies have used two scalar methods of assessing self-confidence; Subjective Technical Competence (STC) used by Arning and Ziefle (2007) and the Affinity to Technology scale by Wolters et al. (2010). Wolters et al. (2010) found that older users who had a high level of affinity to technology had increased rates of task success when using a voice controlled smart home interface. Arning and Ziefle (2007) found a strong association between STC and completion of a task effectively and efficiently amongst the older participant group. Older people reported significantly lower levels of confidence compared with the younger participants of their study. This study aims to investigate whether the self-reported technical confidence of older people influenced the task success rate when using a novel heating control interface prototype. It was hypothesised that older people who reported high technical confidence would have successful interactions with the prototype system.

\section{Method}

This study tested a prototype heating control with a group of older adults who gave their informed consent to the study and received no payment or reward for taking part. The Brunel University School of Engineering and Design Ethics Committee approved the study. Firstly, the study collected demographic data and participants answered a set of paper based technical self-confidence questions. The participants were then 
asked to interact with the prototype by programming some example settings and their success was noted. Once collected the technical self-confidence scores were compared to both task success and other demographic factors.

There were 16 participants aged 52-78 $($ mean $=68.6$ years, male $=7$, female $=9)$, who were recruited through the Brunel Older People Research Group. Of the participants, 12 users said they currently had a digital programmable thermostat at home, however this should be treated with caution. Peffer et al. (2011) cited large inaccuracies in reporting whether households had a manual or programmable thermostats as the terms can cause confusion amongst lay users (Energy Information Administration 2010 and 2011).

\subsection{Technical Self-confidence}

The technical self-confidence questions were based on the Subjective Technical Competence (STC) scale used by Arning and Ziefle (2007) and the Affinity to Technology scale used by Wolters et al. (2010). The STC scale used by Arning and Ziefle (2007 \& 2009) is a shortened version of the original German questionnaire from Beier (1999) containing eight statements. The participant then rated the statements from 1 to 5 as to whether they totally disagree (1) or totally agree (5), giving them a maximum score out of 40 . The Affinity to Technology scale used by Wolters et al. (2010) was tailored to home appliances and voice control as an interaction style. Of the 10 statements 7 more generic statements could be applicable for this study. Of these statements, 3 are a negative version of the previous statement to highlight general disinterest with technology. The statements the participants were asked to rate were:

1. Technology has always fascinated me

2. I really like to try out new gadgets

3. I successfully cope with technical problems

4. Even if problems occur, I continue working on technical problems

5. I really enjoy solving technical problems

6. Up to now I managed to solve most of the technical problems, and I am not afraid of technical problems in future

7. I feel uncomfortable and helpless about using technical devices

8. Technical devices are often not transparent and difficult to handle

9. When I solve a technical problem successfully, it mostly happens by chance

10. Most technical problems are too complicated for me to deal with

\subsection{Usability Task}

The usability task, shown in Table 1, involved programming the control prototype and it was noted whether the participants were successful, used the help features, and any points of confusion during the interaction. 
Table 1. Usability Task Settings

\begin{tabular}{|c|c|c|c|}
\hline Day & Time (12 hour clock) & Time (24 hour clock) & Temperature \\
\hline Monday - Friday & $7 \mathrm{am}-9 \mathrm{am}$ & $07: 00-09: 00$ & $19^{\circ} \mathrm{C}$ \\
\hline & $4 \mathrm{pm}-11 \mathrm{pm}$ & $16: 00-23: 00$ & $21^{\circ} \mathrm{C}$ \\
\hline Saturday \& Sunday & $7 \mathrm{am}-9 \mathrm{am}$ & $07: 00-09: 00$ & $19^{\circ} \mathrm{C}$ \\
\hline & $6 \mathrm{pm}-10.30 \mathrm{pm}$ & $18: 00-22: 30$ & $21^{\circ} \mathrm{C}$ \\
\hline
\end{tabular}

\subsection{Data Analysis}

For the analysis of the results, two statistical methods were used to establish significance. Observed user exclusion was compared to expected exclusion, based on previous analysis published in Combe et al. (2012), and was evaluated using Chisquare tests. Correlations were evaluated using Pearson's product-moment correlation coefficient for parametric variables.

\section{$3 \quad$ Results}

Firstly, the results of the usability testing and task success of participants are reported before correlations between technical self-confidence and success and technical selfconfidence and age are explored.

\subsection{Task Success}

In terms of task success, 9 of the 16 older users were able to programme the prototype for two heating periods on the weekdays and two on the weekends. This gave a success rate of $56.3 \%$ for the older participants. Despite the improved success rate, a Chi-square goodness-of-fit test discerned there was still a significant difference between the observed and expected exclusion of the older age group $\left(\mathrm{p}<0.01\right.$ as $\mathrm{X}^{2}=$ 13.204 and $\mathrm{df}=1)$.

\subsection{Technical Self-confidence and Task Success}

Technical self-confidence data was gathered prior to attempting the task using the prototype. Interestingly, older users who rated themselves as most technically selfconfident were not successful with the task. Participants that rated themselves less confident had greater success, with 3 of the 4 participants that rated their technical self-confidence less than $50 \%$ being successful with the task. These participants also performed quicker with an average task time of 3 minutes 40 seconds compared with 6 minutes 28 seconds for users with technical self-confidence scores above $50 \%$. 


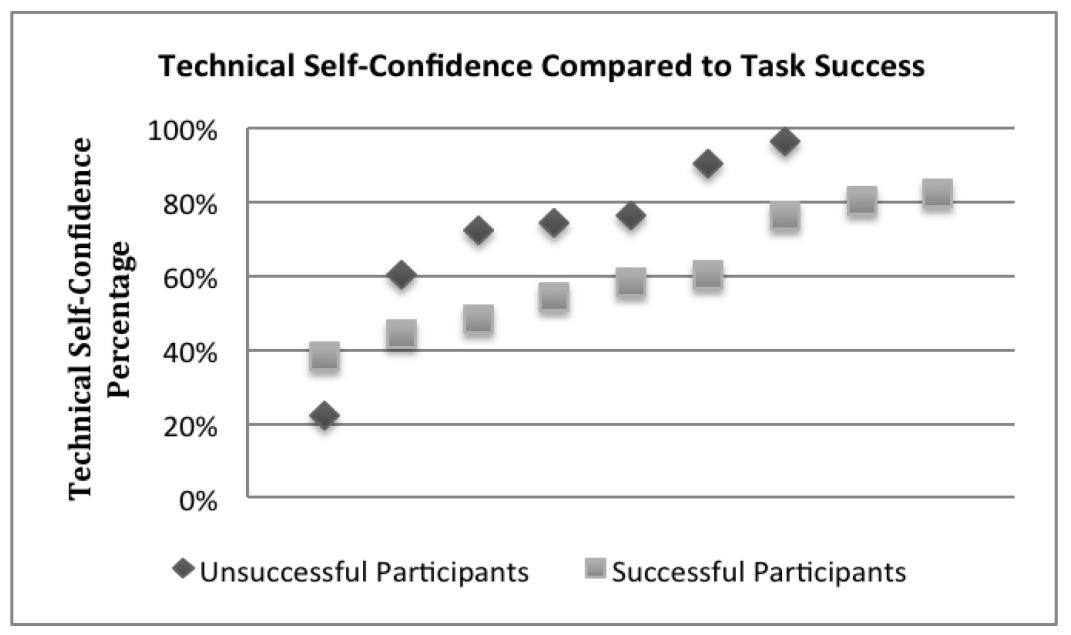

Fig. 1. Task Success vs. Technical Self-Confidence

\subsection{Technical Self-confidence and Age}

Overall, there was a negative correlation between age and technical self-confidence, with self-confidence decreasing with age $(\mathrm{r}=-0.229, \mathrm{n}=31$ and $\mathrm{p}=0.215)$. This negative correlation was small and did not reach levels of statistical significance. Of the unsuccessful older participants, 6 out of 7 rated their self-confidence highly (above 60\%). Participants with a high technical self-confidence appeared to be less patient and got frustrated quickly with the prototype. Highly confident older people were also less tolerant of error messages. Only the participant with the lowest technical self-confidence score of $22 \%$ was also unsuccessful.

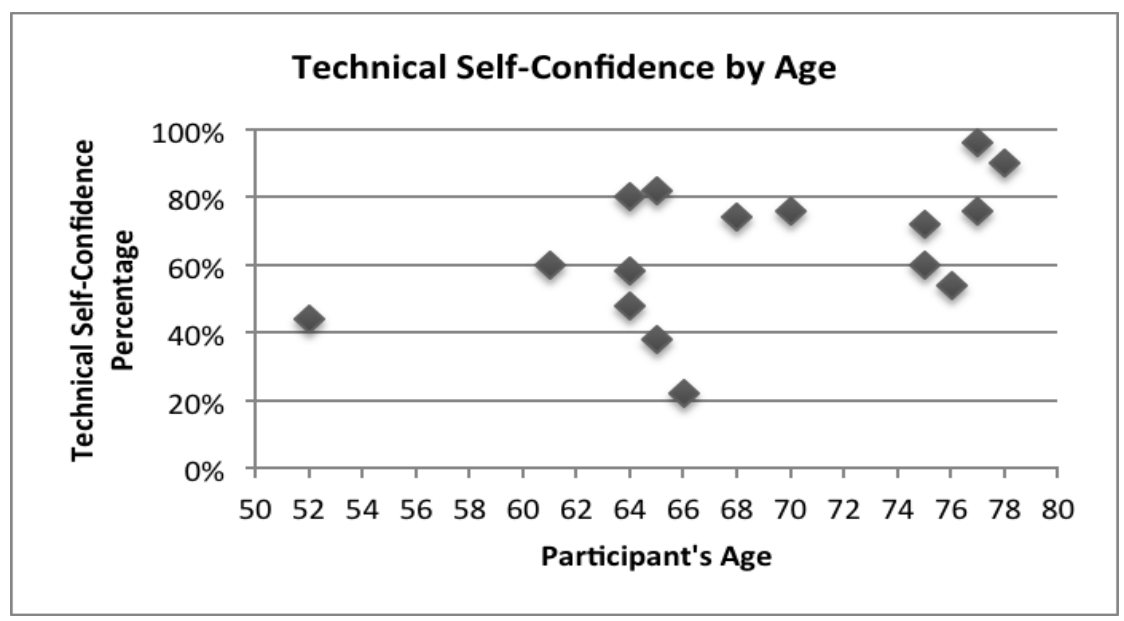

Fig. 2. Age vs. Technical Self-Confidence 


\section{Discussion}

The aim of the study was to investigate whether the self-reported technical confidence of older people influenced the task success rate. Prior experience may have affected task success as only one older participant did not use a computer at all and 13 used a computer on a daily basis. Mobile phone usage varied more. Although all the participants had a mobile phone, only 8 used it on a daily basis and 6 participants used it only to make calls. This technical experience may have contributed to success in the task.

\subsection{Assessing Technical Self-confidence}

Assessing Technical Self-Confidence at the start of the usability testing was a simple and effective way of providing insight into the perception of the user, ahead of the usability testing. Even at a glance it gave the researcher an idea as to how confident the participant was using a technical prototype prior to the testing. This could be useful when including older people in a study sample so that the researcher could support less confident participants in an appropriate manner.

One issue experienced with the application of the scales was some users asked for clarification of the statement "Even if problems occur, I continue working on technical problems". This was considered to be less readable among participants and may be due to the translation for the original German statements. Perhaps this could be reworded for clarity in future use.

Analysis of the completed scales was straightforward, however it was less clear as where the thresholds for high and low confidence should be. This was determined by the researcher as high, over 50\% and low, under 50\% however further work could refine this definition.

\subsection{Limitations and Further Work}

The main limitation of the study was the concept nature of the prototype and the small sample size of the study. The nature of the prototype meant some participants experienced frustration due to limitations with error handling. The participants had high expectations of the performance of the prototype and became frustrated when the prototype did not meet these expectations.

Further work would be required with larger study samples to examine whether this inverse correlation between technical self-confidence and task success is observed with other products or interfaces or is this result an anomaly.

\section{Conclusions}

The negative correlation between reported technical confidence and task success was unexpected, yet very interesting. This is in contrast to the high reliability of the use of such scales reported by both Beier (1999) and Arning and Ziefle (2009). The reported 
confidence of the older people was generally high and highlights that older people expect to be able to interact with systems successfully.

Designing technical systems which give older people positive experiences may improve their technical confidence overall with other systems. With a rapidly ageing population in the UK there is a growing market for technological products, which are both usable and inclusive of older users' needs.

The reported high perceptions of confidence amongst older people should challenge designers' perceptions of older people. This is applicable in the context of both users of technological products and willing participants in usability studies. For designers it would be useful to examine the factors of interactions: which affect the confidence of older people? This leads to the further research questions: does reducing exclusion of older users increase their technical self-confidence? And what design factors can instil confidence within older people?

Acknowledgements. The authors would like to acknowledge both the ESPRC and Buro Happold, who funded this research, and the participants who gave up their time to take part in the study.

\section{References}

1. Abascal, J., Nicolle, C.: Moving towards inclusive design guidelines for socially and ethically aware HCI. Interacting with Computers 17(5), 484-505 (2005), doi:10.1016/j.intcom.2005.03.002

2. Arning, K., Ziefle, M.: Effects of age, cognitive and personal factors on PDA menu navigation. Behaviour \& Information Technology 28(3), 251-268 (2009)

3. Arning, K., Ziefle, M.: Understanding age differences in PDA acceptance and performance. Computers in Human Behaviour 23(2007), 2904-2927 (2007)

4. Beier, G.: Locus of control when interacting with technology. Report Psychologie 24, 684-693 (1999)

5. Caird, S., Roy, R.: User-Centred Improvements to Energy Efficiency Products and Renewable Energy Systems: Research on Household Adoption and Use. International Journal of Innovation Management 12(3), 327-355 (2008)

6. Coleman, R.: Living Longer. In: Clarkson, J., Coleman, R., Keates, S., Lebbon, C. (eds.) Inclusive Design: Design for the Whole Population, 1st edn., pp. 120-142. Springer, London (2003)

7. Combe, N., Harrison, D., Craig, S., Young, M.S.: An investigation into usability and exclusivity issues of digital programmable thermostats. Journal of Engineering Design 23(5), 401-417 (2012), doi:10.1080/09544828.2011.599027

8. Etchell, L., Girdlestone, N., Yelding, D.: Taking control: a guide to buying and upgrading central heating controls, London (2004), http://www.ricability.org.uk/ consumer_reports/at_home/Taking_control/ (accessed on February 28, 2012) (retrieved)

9. Freudenthal, H.J., Mook, A.: The evaluation of an innovative intelligent thermostat interface: universal usability and age differences. Cognition, Technology and Work 5, 5566 (2003), doi:10.1007/s10111-002-0115-6 
10. Langdon, P., Thimbleby, H.: Inclusion and interaction: Designing interaction for inclusive populations. Interacting with Computers 22(6), 439-448 (2010), doi:10.1016/j.intcom.2010.08.007

11. Meier, A., Aragon, C., Peffer, T., Perry, D., Pritoni, M.: Usability of residential thermostats: Preliminary investigations. Building and Environment 46(10), 1891-1898 (2011), doi:10.1016/j.buildenv.2011.03.009

12. Peffer, T., Pritoni, M., Meier, A., Aragon, C., Perry, D.: How People Use Thermostats in Homes: A Review. Building and Environment 46, 2529-2541 (2011), doi:10.1016/j.buildenv.2011.06.002

13. Sauer, J., Wastel, D.G., Schmeink, C.: Designing for the home: A comparative study of support aids for central heating systems. Applied Ergonomics 40, 165-174 (2009)

14. Wolters, K.M., Engelbrecht, K.-P., Gödde, F., Möller, S., Naumann, A., Schleicher, R.: Making it easier for older people to talk to smart homes: the effect of early help prompts. Universal Access in the Information Society 9(4), 311-325 (2010), doi:10.1007/s10209009-0184-x

15. Zhang, B., Rau, P.P., Salvendy, G.: Design and evaluation of smart home user interface: effects of age, tasks and intelligence level. Behaviour and Information Technology 28(3), 239-249 (2009) 